\title{
Calibration and Imaging of CT System Parameters
}

\author{
Yuping $\mathrm{Li}^{*}$, Xianhong $\mathrm{Xu}$, and Zhe Lyu \\ School of Mathematics and Statistics, Zhengzhou Normal University, Zhengzhou, 450044, China
}

\begin{abstract}
The progress of human society cannot be separated from medical development. CT is an important auxiliary instrument of modern medicine, and its imaging precision and stability are essential conditions. To provide a more accurate reference for medicine, data fitting, parameter estimation, interpolation, neural network, continuous discretization, and image processing algorithms are applied in this paper to establish a corresponding model between absorption strength and images through Radon transform and Iradon transform, thereby demonstrating the CT imaging process and CT parameter calibration process. Meanwhile, the CT imaging rules are obtained. As a result, CT parameter calibration precision and stability are improved, as well as diagnosis precision. First, the Excel form is compressed according to the data in Annex 1, and the characteristics of data in Annex 2 are observed. Horizontal illumination and vertical illumination of light are taken to determine the detector unit space. When horizontal light illuminates the medium, the chord passing through the center of the circle is longest. The energy magnitude, physical relation between energy and thickness, and length of the corresponding chord can be found in Annex 2 to establish the matrix equation set and solve the center of rotation of the CT system. The data in Annex 2 corresponds to 180 directions, and the equation set is established to gain every direction.
\end{abstract}

Keywords: center of rotation; imaging precision; stability; Iradon transform energy; X-ray absorptivity

(Submitted on May 5, 2018; Revised on June 19, 2018; Accepted on July 21, 2018)

(C) 2018 Totem Publisher, Inc. All rights reserved.

\section{Introduction}

CT (Computed Tomography) can conduct tomography for biological tissues and engineer material samples according to absorption characteristics of samples for ray energy, in order to gain internal structure information of samples. A typical 2D CT system is shown in Figure 1. X-rays with parallel incidence are perpendicular to the plane of the detector. Each detector unit is deemed as a receiving point and arranged isometrically. The relative position of the X-ray transmitter and detector is fixed. The whole transmission-reception system contra-rotates 180 times around a fixed center of rotation. For every X-ray direction, the ray energy of a tested 2D medium with fixed position after attenuation by absorption is measured on the detector with 512 isometric units, and 180 groups of reception information are obtained after gain processing.

Errors often occur in the installation of the CT system, thus influencing imaging quality. Therefore, parameter calibration is required for the installed CT system. In other words, CT system parameters are calibrated using the samples with known structure (referred to as the template). On this basis, imaging is conducted for the samples with unknown structure.

\section{Modeling}

The 2D calibration template and CT system operation form are shown in Figure 1.

Since there are 512 isometric units on the detector, it is roughly believed that when a light source (expressed as "e") passes through the right circle, a light source (expressed as "f") is parallel to the light source e and passes through the central position of the ellipse. When the light source e passes through the center of the circle, the information received by all light sources passing through the circle reaches a maximum [1].

\footnotetext{
* Corresponding author.

E-mail address: liyuping970301@ sina.com
} 


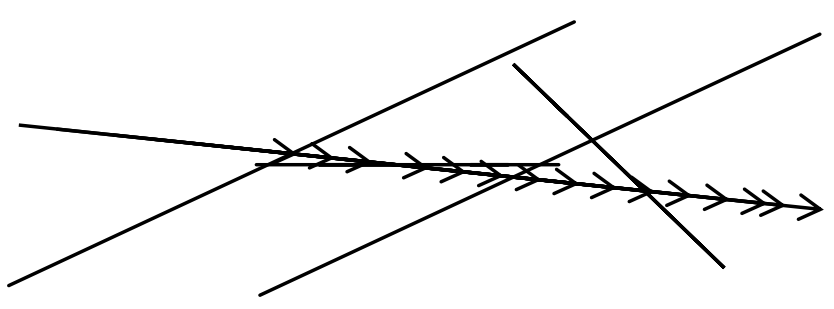

Figure 1. 2D CT operation diagram

From observing the data in Annex 2, we see that the data in the first 13 lines have the following characteristic: only some light sources pass through the circle. Therefore, we study the first line of data conforming to the characteristics and establish a rectangular plane coordinate system based on mutual relations to solve the linear equation of light source e. The slope of straight line $\mathrm{e}$ is the slope of the detector at the initial position of rotation.

Through data analysis, the positions of light source passing through the ellipse, circle, and the middle blank part can be found. Through data observation, we can find that the light source only passes through the cell of the circle, and there is a total of $r$ cells passing through the circle. The diameter length of the circle on the square template is $\mathrm{t} \mathrm{mm}$.

The distance among all detector units is:

Based on the above analysis, we establish Model 1:

$$
I=t / \mathrm{r}
$$

When the light source e passes through $\odot \mathrm{A}$, the maximum value of information received by the light source for the circle is $h_{1}$; the maximum value of information received by the light source for the ellipse is $h_{2}$; the length of the chord intercepted by the light source passing through the ellipse is $\mathrm{n}$; and the radius of the circle is $\mathrm{m}$. Because the ellipse and the circle are on the same template, the respective plane distance and corresponding maximum value proportion of information received are equal. In addition, the length of the major semi-axis of the ellipse is a, and the length of the minor semi-axis is b. Therefore, we can obtain the following:

$$
2 m / h_{1}=n / h_{2}
$$

Thus,

$$
n=2 m h_{2} / h_{1}
$$

The rectangular coordinate system is established based on the square template. The graphs of the ellipse and circle in the rectangular coordinate system are shown in Figure 2.

We suppose the equation of the light source b passing through the center of the ellipse $(c, d)$ is:

$$
x=k(y-d)+c
$$

The equation of the ellipse with a center at $(c, d)$ is:

$$
(x-c)^{2} / b^{2}+(y-d)^{2} / a^{2}=1
$$

Equation (2) and Equation (3) are combined to get:

$$
\left(a^{2} k^{2}+b^{2}\right) y^{2}-2 d\left(a^{2} k^{2}+b^{2}\right) y+d^{2} a^{2} k^{2}+b^{2} d^{2}-a^{2} b^{2}=0
$$

According to the Vieta theorem, we can obtain: 


$$
\begin{gathered}
y_{1}+y_{2}=2 d \\
y_{1} y_{2}=\frac{d^{2} a^{2} k^{2}+b^{2} d^{2}-a^{2} b^{2}}{a^{2} k^{2}+b^{2}}
\end{gathered}
$$

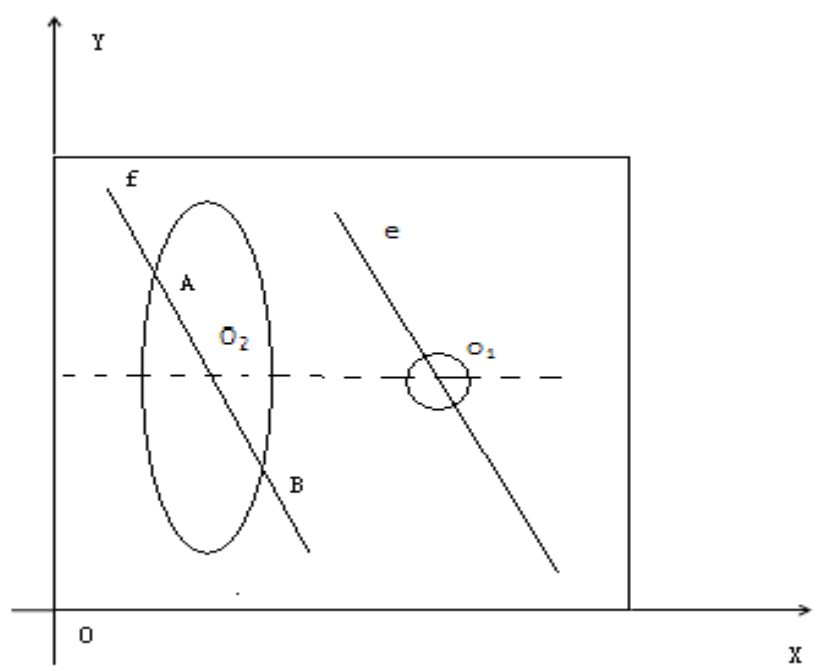

Figure 2. Rays passing through the centers of ellipse and circle

Thus, the chord length is:

$$
|A B|=\sqrt{k^{2}+1} \cdot \sqrt{\left(y_{1}^{2}+y_{2}^{2}\right)-4 y_{1} y_{2}}=2 \sqrt{a^{2} b^{2}\left(k^{2}+1\right) /\left(a^{2} k^{2}+b^{2}\right)}
$$

Since the chord length is n, we can obtain the following, according to $|A B|=n$ :

$$
1 /|k|=\sqrt{\left(4 a^{2} b^{2}-n^{2} a^{2}\right) /\left(n^{2} b^{2}-4 a^{2} b^{2}\right)}
$$

Therefore, the slope of the straight line is:

$$
1 /|k|=\sqrt{\left(4 a^{2} b^{2}-n^{2} a^{2}\right) /\left(n^{2} b^{2}-4 a^{2} b^{2}\right)}
$$

Since the CT system rotates around the center of rotation, a light source 11 always rotates around the center of rotation. According to the above formula, we can find the slope of any straight line passing through the center of the ellipse and then determine the equation of the straight line. According to the distance between cells, we can figure out the distance between 1 and 11. Then, according to the relation of parallel lines, the equation of 1 can be solved. Then, two lines can be determined. Since 1 rotates around a fixed point, the intersection point of three lines is the center of rotation of the CT system [2].

By drawing data from Appendix 3 in MATLAB, the following figures can be obtained:

By using MATLAB to overlap and contrast the energy cross section of Annex 2 and Annex 3, it is found that the image of the Annex 3 medium is basically similar to the outline of the image of Annex 2. Thus, the outline of the media figure corresponding to the data of Annex 3 is generally an ellipse.

Then, the number of more than 0 of the data of Annex 2 and Annex 3 is 1, and the number of the maximum values in the sum data is found using the total sum of the collected data. The number of columns corresponding to the maximum value of Appendix 2 after the summation is basically the same as the number of maximum values in Annex 3 . The number of columns corresponding to the minimum value of Appendix 2 after the summation is basically the same as the number of minimum values in Annex 3, the initial direction of the X-ray obtained by the problem (I), and the rotation angle. The 
following are combined: the number of maximum corresponding columns of Annex 2 and Annex 3, the number of times of rotation, and the number of the approximate coincidence of Appendix 2 and Annex 3, which is the horizontal and vertical direction of the X-ray in the CT system [3].

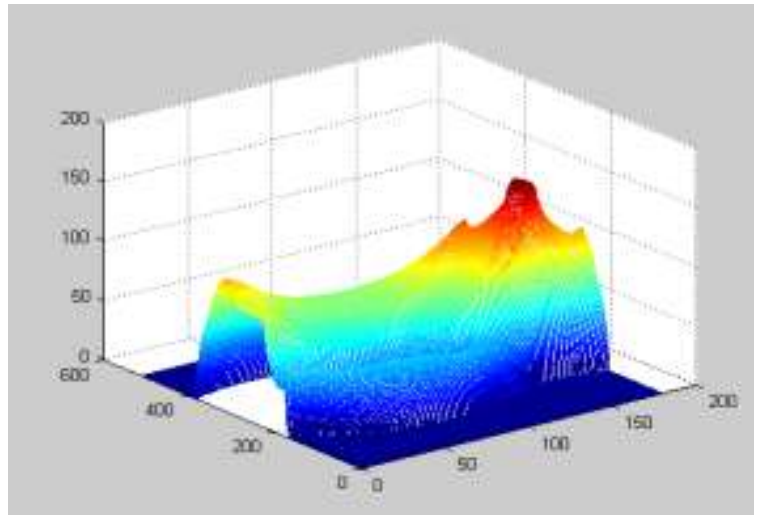

Figure 3. Rbsorption energy diagram of medium

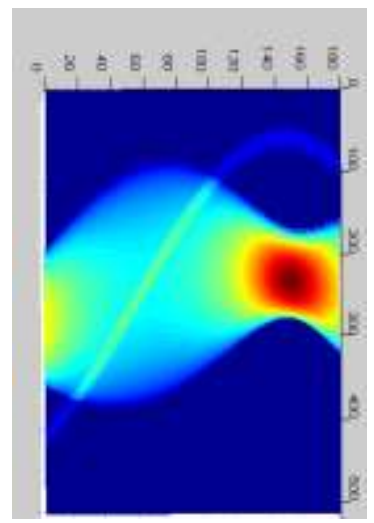

Figure 4. Energy absorption cross section of Annex 2 and Annex 3

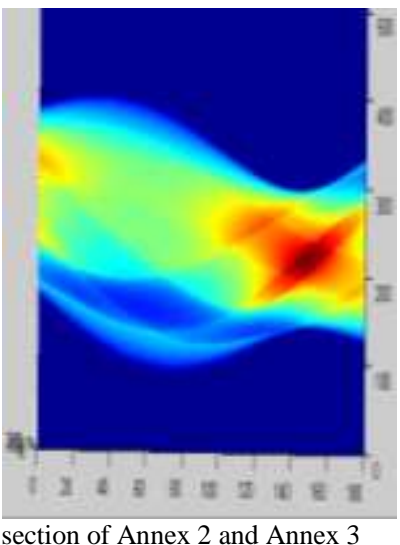

It can also be explained that the graph outline corresponding to the data given in Annex 3 is roughly an ellipse.

Then, according to the cell corresponding to the source of the rotation center in Model 1, we find the cell that corresponds to the maximum value of the receiving information according to the data of the cell in Annex 3 . Then, the cell is at the center position of the corresponding figure in Annex 3.

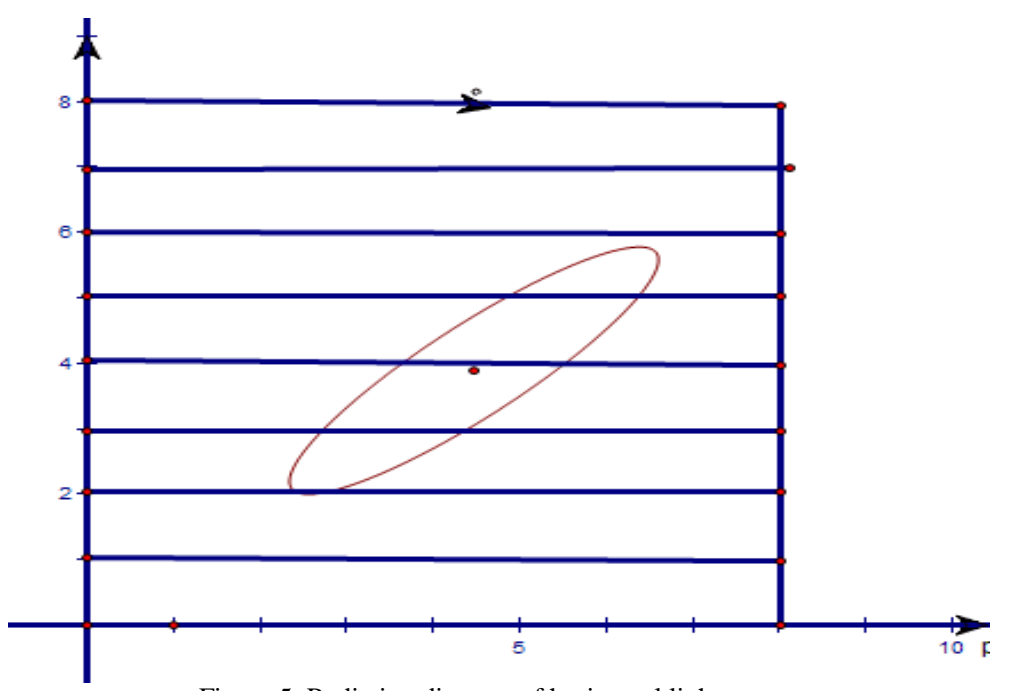

Figure 5. Radiation diagram of horizontal light source

The distance between the two cells can also be solved according to the formula in Model 1. The two straight lines are parallel. According to the distance between the two lines, we can obtain the vertical coordinate value of the graphic center. In the same way, we can find the transverse coordinates of the graphic center. At this time, the corresponding figures in Annex 3 can be obtained in the coordinate system based on the rectangular coordinate system, with the template as the reference.

\section{Model Solving}

According to the data in Annex 1 and Annex 2, we can get:

First of all, isometric compression of the data in Annex 1 is conducted to gain the following Figure 6: 


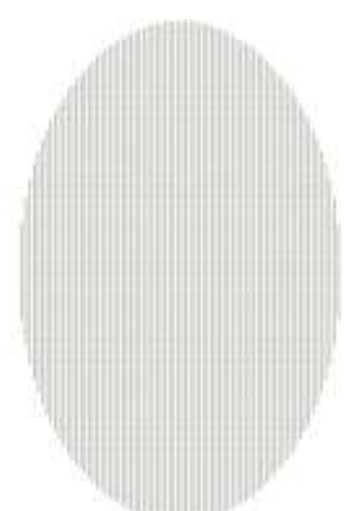

Figure 6. Isometric compression of the data

For Annex 2, MATLAB is applied for drawing, and the following figure is obtained:

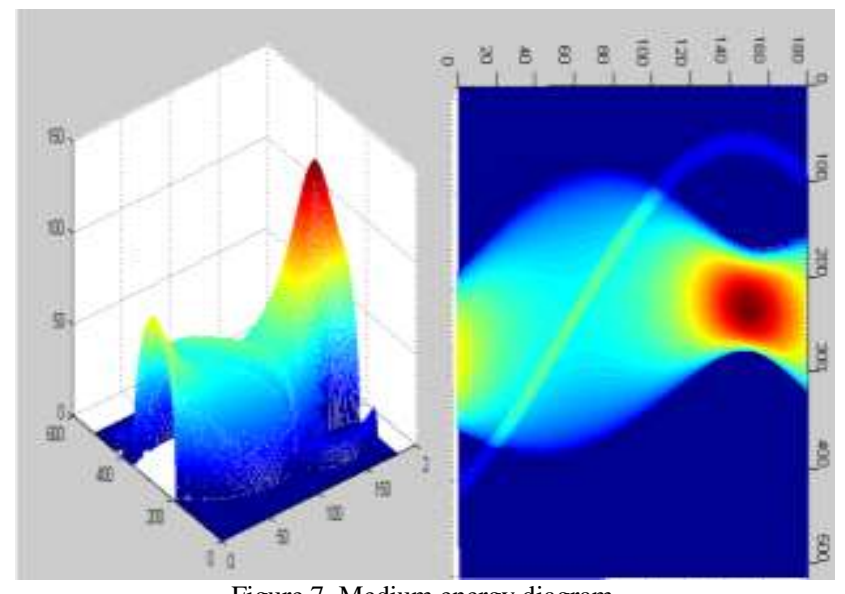

Figure 7. Medium energy diagram

According to the data analysis in Annex 2, the positions of the light source passing through the ellipse, circle, and middle blank part can be determined. Through data observation, we can conclude that the data from A to $\mathrm{M}$ belong to the cells where the light source passes through the circle, and a total of 29 cells pass through the circle. The diameter length of the circle on the square template is $8 \mathrm{~mm}[4]$.

Thus, the distance between detector units is:

$$
\begin{gathered}
I=8 / 29=0.2759 \\
I(R, E)=I_{0}(E) e^{-\int_{0}^{R} u(E) \mathrm{d} x} \\
I=I_{0} e^{-u L}
\end{gathered}
$$

The values required can be gained through the first line of data conforming to the characteristics and the data in Figure 1 and Figure 2.

In the first line, the maximum value of information received by the circle is $h_{1}=14.1766$; the maximum value of information received by the ellipse is $h_{2}=89.7648$; and the radius of the circle is $m=4$. The length of the major semi-axis of the ellipse is $a=$, and the length of the minor semi-axis is $b=$. The center of the ellipse is at $(50,50)$, and the center of the circle is at $(95,50)$.

The data are substituted into the equation of Model 1, with $n=50.6552$. Then, we get $|k|=0.5689$. 
The equation slope of the straight line passing through the center of the ellipse and the center of the circle is $1 / k=-1.7578$. In addition, the corresponding straight line slope of the parallel light is the initial direction of detector rotation. Then, the included angle between the direction of light source and positive $\mathrm{x}$-axis is $\alpha=119.6353$.

In accordance with the last line of data in Annex 2, the maximum value of information received by the circle is $h_{1}$ $=14.1772, h_{2}=91.4616$.

$$
n=2 m h_{2} / h_{1}=2 \times 4 \times 91.4616 / 14.1772=51.3501
$$

So,

$$
\frac{1}{|k|}=\sqrt{\frac{4 \times 40^{2} \times 15^{2}-51.3501^{2} \times 40^{2}}{51.3501^{2} \times 15^{2}-4 \times 40^{2} \times 15^{2}}}=1.8321
$$

The end direction of detector rotation is a straight line with a slope of -1.8321. At this moment, the included angle between the direction of light source and positive $\mathrm{x}$-axis is $\alpha=119.6353$.

Thus, the equation for the straight line is

$$
e_{1}: \quad y=-1.7578 x+137.89
$$

We suppose the center of rotation is at $(\mathrm{x} 1, \mathrm{y} 1)$. The straight line passing through the center of rotation is $y_{1}=k_{1} x_{1}+b_{1}$. The cell corresponding to the center of rotation is $z$. Thus, we can obtain the equation for a straight line parallel to e1 and passing through the center of rotation as

$$
y=\frac{-1.7578 x+137.89+((274-z) \times 0.2759 \times(-1.7578))}{1 / \sqrt{1+(-1.7578)^{2}}}
$$

Then, we choose the DU line of data. Through the same calculation, we can obtain the equation for a straight line passing through the center of rotation $(\mathrm{x} 1, \mathrm{y} 1)$ with a slope of $\mathrm{k} 1=1.5200$ :

$$
y=\frac{1.5200 x-26+((216-z) \times 0.2759 \times 1.5200)}{1 / \sqrt{1+1.5200^{2}}}
$$

Then, we choose the FX line of data. Through the same calculation, we can obtain the equation for a straight line passing through the center of rotation $(\mathrm{x} 1, \mathrm{y} 1)$ with a slope of $\mathrm{k} 1=-1.8402$ :

$$
y=\frac{-1.8321 x+141.615+((238-z) \times 0.2759 \times(-1.8321))}{1 / \sqrt{1+(-1.8321)^{2}}}
$$

Equations (5), (6), and (7) are combined to get:

$$
\left\{\begin{array}{l}
x=35.2809 \\
y=57.8985 \\
z=255.6733
\end{array}\right.
$$

Thus, the point of coordinate system $(35.2809,57.8989)$ is established with a unit of $1 \mathrm{~mm}$ when the center of rotation takes the peak of the lower right corner as the original point and the side of the square template as the coordinate axis.

The position of the CT center of rotation in the square tray is as follows: the point in the coordinate system is 
established with a unit of $1 \mathrm{~mm}$, the peak of the lower right corner of the template serves as the original point, the side of the square template serves as the coordinate axis, and the point coordinates are $(35.2809,57.8989)$. The distance between detector units is $\mathrm{I}=0.2759$. The scope of the included angle of 180 directions used in the CT system between the direction of light source and positive $\mathrm{x}$-axis is $(-60.36370,118.62410)$. In addition, the interval is divided into 180 parts equally, and the direction corresponding to the angle of each part is the direction of the X-ray [5].

According to the data obtained from the model, the 256th cells is passed through the center of rotation. When the light source rotates in a direction perpendicular to the x-axis, we find a single cell that conforms to the center of the 217 th cell, and the difference between the two cells is:

$$
p_{1}=256-217=39
$$

The distance between the two cells is:

$$
d_{1}=0.2759 \times 39=10.7586
$$

Because the coordinates of the center of rotation are $(35.2809,57.8989)$.

Thus, the transverse coordinates of the graphic center can be obtained as follows:

$$
x_{2}=10.7586+35.2804=46.0935
$$

When the light source rotates in the direction perpendicular to the $y$-axis, the cell of the light source passing through the graphic center is the $243 \mathrm{rd}$ cell, and the difference between the two cells is:

$$
p_{2}=256-243=13
$$

The distance between the two cells is:

$$
d_{2}=0.2759 \times 13=3.5862
$$

Thus, the transverse coordinates of the graphic center can be obtained as follows:

$$
y_{2}=59.8985-3.5862=54.3123
$$

It can be concluded from the above that the center of the approximate ellipse in Annex 3 is located at the point $(46.0935,54.3123)$.

There are many kinds of imaging techniques. The principle of imaging in CT is Radon transform, which can be regarded as a linear integral of a function $f(x, y)$ that is represented by a two-dimensional figure in the plane in a different direction. The value of the linear integral in the different directions of each point in the two-dimensional graph is obtained. The inverse transform of the Radon transform is Iradon transformation, that is, the corresponding 2-D image is determined by the value of the linear integral, and the CT system is the imaging principle [6].

The value in Annex 3 is the value of a linear integral corresponding to a two-dimensional graph. The value of the Iradon function in MAT-LAB can be used to draw a three-dimensional map of the Radon transformation using the value of Annex 3.

As shown in Figure 9, the image absorption rate of the blue part can be seen as 0 , that is, the remaining color of the unabsorbed intensity is the absorption strength of the X-rays passing through various points of the unknown medium, and the different colors indicate the different sizes of the absorption strength. Thus, the specific shape of the unknown medium can be determined as two small elliptical holes on both sides of a large ellipse.

As shown in Figure 9, the unknown medium is not a homogeneous object. The different colors in the diagram are of different absorption intensity (absorption rate), and the data in Annex III are processed by the gain (i.e., data amplification). The original absorption rate is $\mathrm{Q}$, and the absorption rate after gain treatment is $\mathrm{Q}$. The relationship between the two is $\mathrm{Q}=$ theta $\mathrm{Q}$. 


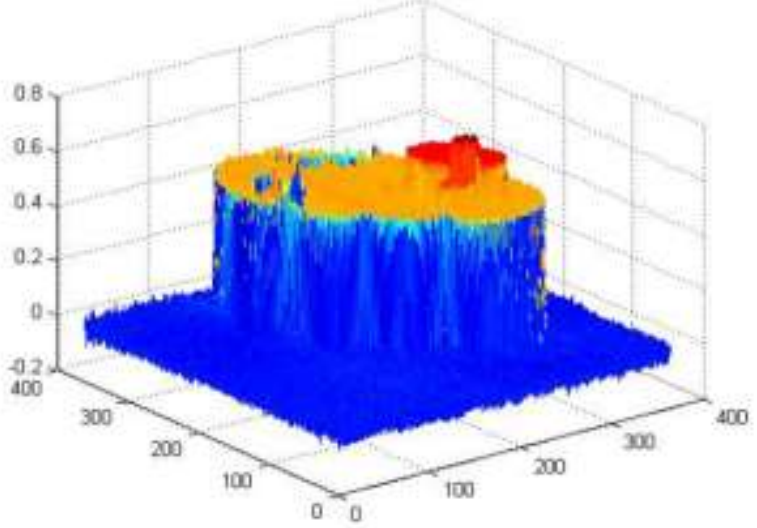

Figure 8. Radon transform 3D diagram

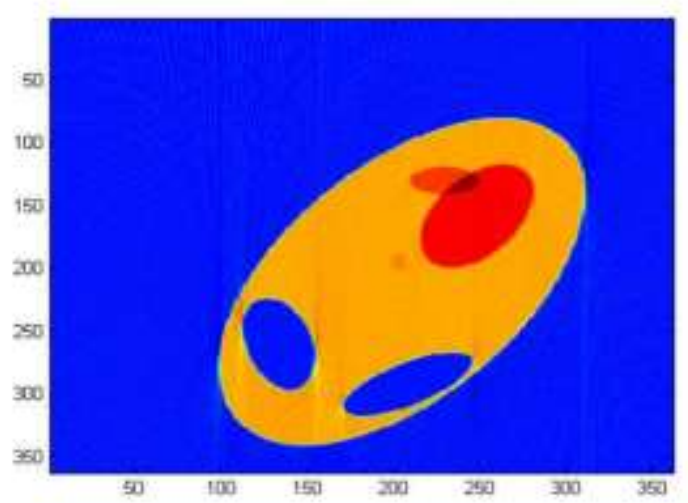

Figure 9. Radon transformation plane schematic diagram

When a beam of light is perpendicular to the $\mathrm{X}$ axis, the absorption rate is $\mathrm{Q}=20$ when a beam of light passes through the long axis of the ellipse. The absorption rate of this point corresponds to the ray energy $\mathrm{E}=14.1769$ in the same direction as Annex 2, assuming the energy and absorptivity are related such that $\mathrm{E}=[$ lambda]Q can obtain [lambda] $=0.7088$. We can transform the data in Annex II, divide it by lambda, and obtain the data of the absorption rate, as Annex 6 [7].

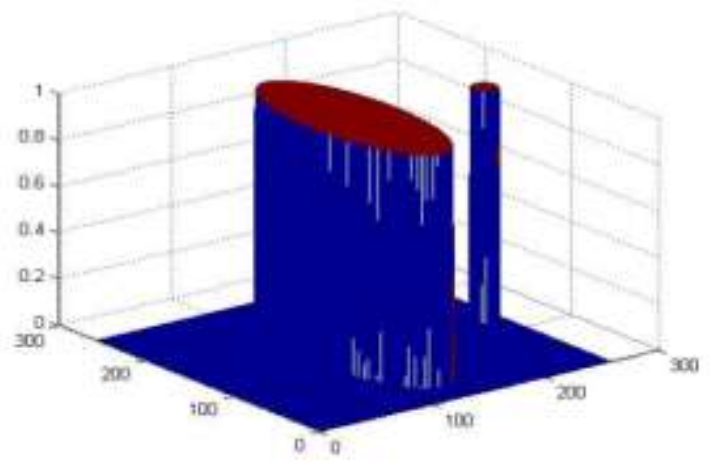

Figure 10. Absorptivity diagram

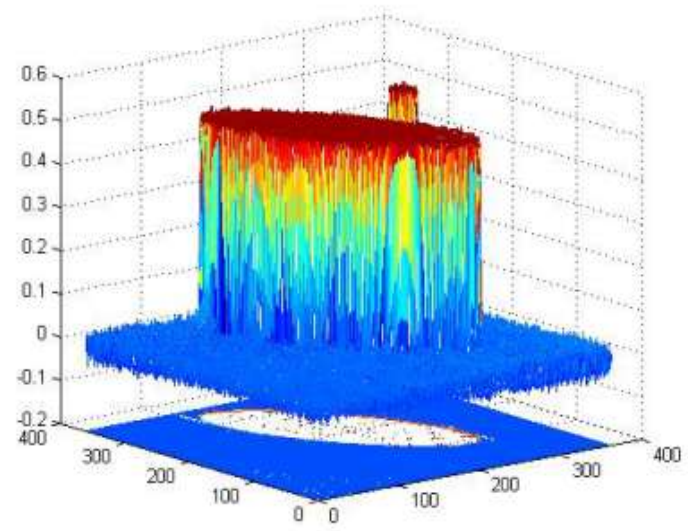

Figure 11. Absorptivity contrast diagram

A schematic diagram of the absorbability of Annex 1:

Data from Annexes 1 and 6 can be drawn below:

It can be seen from the graph that $q=1$ and $Q=0.5$, so lambda=2. 
The approximate distribution of absorptivity in the unknown medium is as follows [8]:

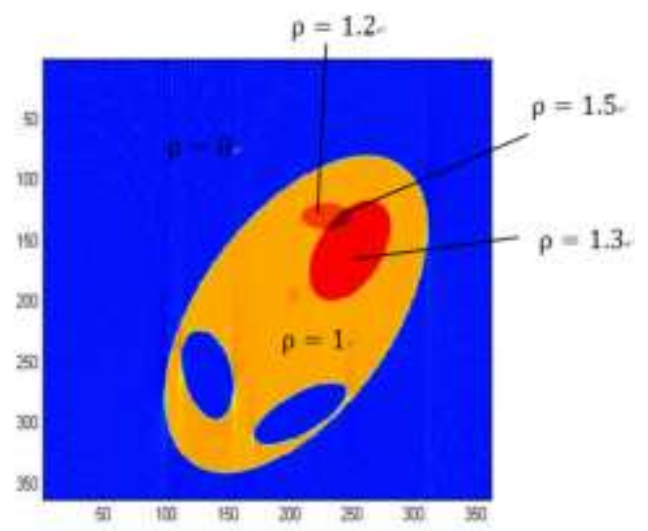

Figure 12. Absorptivity distribution diagram

\section{Improved Model}

In (1), a contrastive analysis is carried out for Annex 1 and Annex 2. Then, MATLAB is used for data analysis. Several groups of special values are taken for analysis and calculation. Parameter calibration is conducted in (1). However, data selection is single (e.g., there is certain error). When the light passes through the small circle, after the maximum data of each line is taken from the data of the small circle in Annex 2, the mean value is solved. Because the maximum value when the light illuminates the small circle corresponds to the diameter of the small circle, the specific value I can be determined, i.e., the relation of absorptive amount per unit length.

$$
I=\frac{2 m}{\left(q_{1}+q_{2}+q_{3}+L q_{i}\right) / n}, \quad i \in N^{+}
$$

Then, the lines when the ellipse is illuminated alone and the maximum value of energy absorbed by the line are taken. The following can be solved by the relation between length and absorptive amount.

$$
n_{j}=\frac{2 m p_{j}}{\left(q_{1}+q_{2}+q_{3}+L q_{n}\right) / n}, \quad j \in N^{+}
$$

Then, multiple $n_{j}$ are solved, and the maximum value is substituted into Formula (4). Because the equation passes through one point $(c, d)$, multiple equations can be gained. The center of rotation corresponding to multiple groups of data is solved. Then, the multiple groups of data are fitted to determine the accurate center of rotation. Finally, the position of the tray where the medium is can be obtained [9].

Through data analysis, some more accurate installation positions can be gained.

The center of rotation of the CT system, the central position of the tray where the medium is put, and the central position of the medium in the diagram are accurately sought, and the three points are superposed. At this moment, the error of rotation is relatively small.

\section{Model Evaluation}

\subsection{Advantages}

- It is simple, easy to solve, and understands the parameters of the CT system calibrated by a particular value.

- Relevant information of an unknown medium image is confirmed according to calibration parameters. The application scope of the Radon inverse operation is wide, so it can be promoted. 


\subsection{Disadvantages}

- The data used in calculations are limited, and not all characteristics will be completely reflected. The accurate fitting calculation cannot be completed for the data.

- When the CY system rotates, two light sources pass through the center of the ellipse and circle, which cannot be accurately confirmed [10].

\section{Acknowledgements}

This paper was partly supported by the Fund Program and the Key Program of Zhengzhou Normal University (2016JSJGZD-014).

\section{References}

1. C. Zhang, "Industrial CT Technology and Principle," Science Press, pp. 529-551, April 2009

2. C. Ma, "Research on Key Technology of CT Image Reconstruction," PLA Information Engineering University, pp. 45-46, December 2011

3. C. Peng and Z. Li, "Influence of Positional Accuracy of Wu Haifeng Ray Source on Industrial CT Images," Atomic Energy Science and Technology, pp. 15-19, January 2004

4. D. F. Wu, L. Li, L. Zhang, Y. X. Xing, Z. Q. Chen, and Y. S. Xiao, "Geometric Calibration of Cone-beam CT with a Flat-panel Detector," in Proceedings of 2011 IEEE Nuclear Science Symposium Conference Record, pp. 2952-2955, 2011

5. J. Hu, J. Zou, J. Gui, J. Rong, and Q. Zhang, "Geometric Calibration based on Identification of Ellipse Parameters of a MicroCT System for Small-Animal Imaging,” Vol. 9, No. 5, pp. 1938-1942, October 2011

6. M. Daly, J. Y. Siewerdsen, D. Jaffray, and J. Irish, "Geometric Calibration of a Mobile C-arm for Intraoperative Cone-beam CT,” Medical Physics, Vol. 35, No. 5, pp. 2124-2136, 2008

7. S. Wang, "Mathematical Modeling," Beijing: Science Press, pp. 107-109, March 2008

8. X. Qian, "Study on Geometrical Parameter Correction Method of Cone Beam CT," Chongqing University, pp. 5-7, May 2016

9. X. U. Yang, "Parameter Calibration in Imaging of CT System," in Proceedings of $20172^{\text {nd }}$ International Conference on Computer Engineering, Information Science and Internet Technology, pp. 58-61, February 2017

10. Y. Song, "Numerical Analysis and Application”, Beijing: China Machine Press, pp.15-20, November 2009

Yuping Li: Master's degree, associate professor, research direction in mathematical modelling.

Xianhong Xu: Ph.D., lecturer, research direction in industrial statistics.

Zhe Lyu: Master's degree, assistant lecturer, research direction in education statistics. 